\title{
High Precision Spectrometers for Very Forward Protons in CMS
}

\author{
Michael G. Albrow \\ Fermi National Accelerator Laboratory, Batavia, IL 60510, USA
}

\begin{abstract}
We plan to add proton tracking and timing detectors at $z=240-250 \mathrm{~m}$ on both sides of CMS to study central exclusive production, with one or both protons measured, and single diffraction. They provide measurements of $p+p \rightarrow p+X+p$, where $X=Z, H, W^{+} W^{-}$and multiparticle states (with or without jets), as well as single high mass diffraction in low pile-up runs.
\end{abstract}

Keywords: Higgs, Central exclusive production

PACS: $14.80 . \mathrm{Ec}, 27.75 . \mathrm{Dw}$

Plans to add very forward proton detectors at $z= \pm 420 \mathrm{~m}$ from ATLAS and CMS, called FP420, to measure exclusive ${ }^{1}$ Higgs boson production, $p+p \rightarrow p+H+p$ were developed starting in 2003 in a series of "Manchester Meetings" [1], following ideas developed in 1998-2000 [2, 3, 4]. The cross section is in reach at the LHC if high luminosity, $\mathrm{L} \sim 10^{34} \mathrm{~cm}^{-2} \mathrm{~s}^{-1}$, with $\sim 25$ collisions per bunch crossing every $25 \mathrm{~ns}$, can be used. This requires [2] precision timing $(\Delta t(p p))$ on the protons to get a vertex $z_{p p}$ to match the $z_{\text {vertex }}$ of the Higgs candidates, which, together with kinematic constraints, can reduce pile-up background to a manageable level. (If good timing is added to the central detectors, additional pile-up rejection is possible.) The forward proton detectors are small, $15(x) \times 12(y) \mathrm{mm}^{2}$, and with silicon pixel detector stacks spaced by $10 \mathrm{~m}$ we can measure the position and angle, $\left(x, \theta_{x}\right)$, to about $(10 \mu \mathrm{m}, 1 \mu \mathrm{rad})$. In order to have acceptance for a $125 \mathrm{GeV}$ Higgs boson, $H(125)$, with both protons measured, there has to be at least one station at $z=420 \mathrm{~m}$ after $120 \mathrm{~m}$ of superconducting dipoles. There is a missing dipole there, and a cryogenic bypass can be installed to expose a roomtemperature beam pipe; also the machine optics is ideal. At this location the deflected protons, with fractional momentum loss $\xi=1-p_{z} / p_{\text {beam }}$, are between the two beam pipes and space is limited, so traditional "Roman pots", as used since the early ISR days, are not possible. We have developed a different type of vacuum chamber, a "moving pipe", initially used by ZEUS at HERA. A $40 \mathrm{~cm}$ section of vacuum pipe has a thin flat wall (pocket) on one side, and when the beams are stable the pipe (between bellows) is moved sideways to bring the detectors within $2-3 \mathrm{~mm}$ of the circulating beam. Unlike Roman pots, there are no differential forces involved, and the pockets can have much more space for the detectors, while being compact in the $x$-direction. Our plan is to have two pockets per beam; the first with silicon pixel tracking detectors, and the second also with timing detectors [5], with $\sim 10$ ps resolution, at the back.

\footnotetext{
${ }^{1}$ Exclusive means no other particles are produced.
} 
A report on the FP420 R\&D project, which was joint venture between both ATLAS and CMS physicists, was published [6] in 2009. Following that period, the ATLAS and CMS groups proceeded semi-independently, calling the proposed subdetectors AFP (ATLAS Forward Protons) and HPS (High Precision Spectrometers) respectively. I report on the HPS. We proposed a two-stage approach: Stage 1 is at $z= \pm 240$ $250 \mathrm{~m}$, where the vacuum pipe is clear of obstruction and installation is straightforward. However the acceptance for $M(X)=125 \mathrm{GeV}$ with both protons detected is small and only at high momentum-transfer squared $-t \gtrsim 4 \mathrm{GeV}^{2}$. In Stage 2 we add stations at 420-430 m, where a cryogenic bypass must be made. In addition to the high acceptance for $\mathrm{H}(125)$ with both protons measured, the $(120 \mathrm{~m} \times 8 \mathrm{~T})$ dipoles between $240 \mathrm{~m}$ and $420 \mathrm{~m}$ give much better momentum resolution. (The magnets upstream of 240 $\mathrm{m}$ are quadrupoles, $28 \mathrm{Tm}$ of warm dipoles to separate the beams, and a $35.9 \mathrm{Tm}$ superconducting dipole to bring the beams parallel.) In Stage 1 the full set of detectors can be made operational and a physics program started with $M(X) \gtrsim 200 \mathrm{GeV}$, and one or two years later the cryogenic bypasses could be installed to complete Stage 2 .

At $240 \mathrm{~m}$ the scattered protons are not between the beam pipes but towards the outer (larger radius) wall, This means that in principle Roman pots can be used there, if the longitudinal $(z)$ space limitation is not an issue ${ }^{2}$. In Stage 2 there is not space.
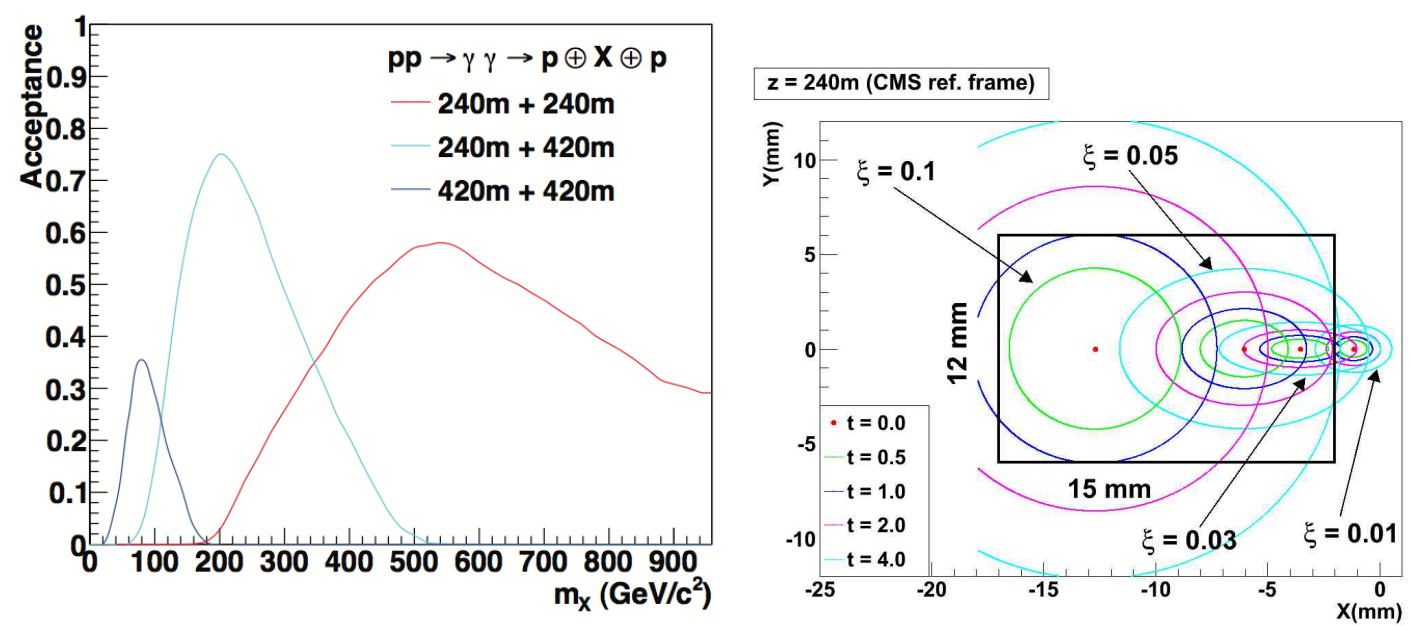

FIGURE 1. Left: Acceptance at small $t$ for both protons detected at different stations. Right: Contours at fixed $t, \xi$ in the $x, y$ plane at $240 \mathrm{~m}$. For any $t, \xi$ the acceptance is approximately the fraction of the ellipse contained in the detector area $(-6 \mathrm{~mm}<y<+6 \mathrm{~mm},-17 \mathrm{~mm}<x<-2 \mathrm{~mm})$.

The acceptance in Stage 1 includes $Z$ and $H(125)$ with one proton measured, but not with both. The acceptance, see Fig. 1 , includes $0^{\circ}$ scattering for $0.015<\xi<0.12$, which allows photon exchange to be detected. The $t$ measurement can be important to distinguish $\gamma$ and $\mathbb{P}$ exchange. These figures are for a detector active edge at $2 \mathrm{~mm}$ from the beam center (at $3 \mathrm{~mm}$ the acceptance is reduced for $\xi \lesssim 0.02$ ). For $p+H(125)+p$

\footnotetext{
2 After this Workshop different scenarios are under evaluation, including the possibility of Stage 1 using Roman pots at $240 \mathrm{~m}$ or $204-216 \mathrm{~m}$.
} 
the best acceptance is with $240+420 \mathrm{~m}$ stations, but the mass resolution is best with $420+420 \mathrm{~m}$. The $240 \mathrm{~m}$ (but not the $420 \mathrm{~m}$ ) detectors are near enough to be included in the level 1 trigger.

I now discuss some physics that can be done in Stage 1 with detector stations at 240 m with both protons measured, $p+X+p$, and acceptance $M(X) \gtrsim 200 \mathrm{GeV}$. Later I consider what may be done with a single proton measured, with acceptance down to lower $M(X)$ but with fewer constraints. The production of a state $X$ between two large rapidity gaps in $p p(p \bar{p})$ can occur through three processes [7]: $\gamma+\gamma(\mathrm{QED}), \gamma+\mathbb{P}$ (photoproduction), and $\mathbb{P}+\mathbb{P}$ (double pomeron exchange). The following processes were observed for the first time (in $p \bar{p}$ ) by CDF: $\gamma \gamma \rightarrow e^{+} e^{-}, \mu^{+} \mu^{-}, \gamma \mathrm{IP} \rightarrow J / \psi, \psi(2 S)$, and IPIP $\rightarrow \chi_{c 0}, \gamma \gamma$, and dijets. At the LHC the higher energy opens up central exclusive production as a new window on electroweak processes, thus: $\gamma+\gamma \rightarrow W^{+} W^{-}, \gamma+\mathrm{IP} \rightarrow$ $Z$, and $\mathbb{P}+\mathbb{P} \rightarrow H$. Stage 1 only has acceptance for the first of these reactions, unless there is a heavier Higgs. We have now discovered a state at $125 \mathrm{GeV}$, and it is important to measure its properties every way we can. Looking like a Standard Model Higgs, it may be an MSSM SUSY $h^{0}$, partnered by a heavier $H^{0}$, with $M>200 \mathrm{GeV}$. A state coupling mostly to fermions has much less background in $\tau^{+} \tau^{-}$than in $t \bar{t}$ and $b \bar{b}$. In inclusive $\tau^{+} \tau^{-}$the mass resolution is poor because of the missing neutrinos, but in exclusive production with $p_{T}\left(\tau^{+} \tau^{-}\right)$small and one or both protons measured, an overall fit can be done and the $M\left(\tau^{+} \tau^{-}\right)$resolution improved to only a few $\mathrm{GeV}$.

The $\gamma \gamma \rightarrow W^{+} W^{-}$reaction is guaranteed, with a SM cross section $\sigma(W W, M>300)$ $\mathrm{GeV} \sim 50 \mathrm{fb}$. This is a factor $\sim 20$ larger than the $\gamma \gamma \rightarrow \mu^{+} \mu^{-}$cross section, because the $t$-channel exchange is $J=1$ rather than $J=\frac{1}{2}$ [7]. The $W$ are transverse and do not access the Higgs sector, but this channel is sensitive [8] to anomalous quartic gauge couplings. In $10 \%$ of the events both $W$ decay leptonically $(e, \mu, \tau)$ so they can be triggered on, the QCD background is absent, and the kinematics fully constrained. Requiring no other tracks within $1 \mathrm{~mm}$ of the dilepton vertex cleans the signal with little loss of efficiency. The cross section $\sigma(\gamma \gamma \rightarrow x \bar{x})$ depends only on the charge, spin and mass of the particle $x$. Although charged sleptons can be pair-produced, the cross section is much too small because the $t$-channel exchange has $J=0$. (On the contrary, a charged $J=2$ particle would have $\sigma \gg \sigma(W W)$.)

Exclusive QCD dijet production, $X=J J$, can be selected under a large background with timing $\left(z_{p p}=z_{X}\right)$, longitudinal momentum balance $\left(p_{z J 1}+p_{z J 2}\right)=-\left(p_{z p 1}+p_{z p 2}\right)$, $\sum_{i=1,2} E_{T, i}=-\sum_{i=1,2} p_{T i}(p) \sim 0$, with the jets opposite in azimuth $\phi$. One can include three jets, and can clean the sample by requiring track isolation, i.e. find all the tracks on the jets' common vertex and calculate their transverse momenta $k_{T}$ with respect to the jet axes. Then select events having no tracks with $k_{T} \gtrsim 1 \mathrm{GeV} / \mathrm{c}$. The $M(J J)$ spectrum itself is a good test of the theory of hard pomeron interactions, which also predicts that $>99 \%$ of these dijets are gluon jets, and the rest are nearly all $b \bar{b}$ jets. This tests the $J_{z}=0$ rule [4] which forbids light quark dijets when the protons have $t \sim t_{\min }$. So it is important to have very efficient $b$-tagging with few fakes, and both protons measured. The exclusive $b \bar{b}$ dijet spectrum should be measured as well as possible, to test QCD and to estimate the background for $H \rightarrow b \bar{b}$.

When only one proton is detected one does not have constraints on the vertex $z$ from timing, or on $M(X)$ from the missing mass to the protons. This will make physics under normal high pile-up conditions very difficult or impossible, except for some particularly 
clean final states such as exclusive $\tau^{+} \tau^{-}, W^{+} W^{-}$or $Z$ with leptonic decays. Single diffractive dijet candidates, with $p$ and $J J$, are likely to be from different events. With the HPS operational we can hope for at least some days of special running with average pile-up $\mu \sim 1$. In 120 hours with 2800 bunches and $\mu=1$ we would have $180 \mathrm{pb}^{-1}$ delivered, and $66 \mathrm{pb}^{-1}$ for single no-pile-up collisions. (Without seeing the protons, about 100 exclusive $\gamma \gamma$ events with $M(\gamma \gamma)>10 \mathrm{GeV}$ could be measured, another test of the exclusive Higgs mechanism.) The single diffractive $Z$ and $W$ cross sections are $\sim 10$ - $100 \mathrm{pb}$. High mass single diffraction, $p+W, Z, J J, Q \bar{Q}$ would be extremely valuable to enhance our understanding of QCD in the diffraction sector.

Consider exclusive $\tau$-pair production: $X=\tau^{+} \tau^{-}$; the $\tau$ 's decay to 1 -track (85\%) or 3 collimated tracks with low mass (15\%); $40 \%$ of the pairs have an $e$ or $\mu$ for a trigger. The $\tau$ 's will have $\Delta \phi \sim \pi$ and that, together with no other tracks on the $\tau$-pair vertex $\left(n_{\text {ass. }}=0\right)$, will reject almost all the Drell-Yan background. Three mechanisms can produce this final state: $\gamma+\gamma, \gamma+\mathbb{P} \rightarrow Z$, and $\mathbb{P}+\mathbb{P} \rightarrow H$. The first two produce $e^{+} e^{-}$ and $\mu^{+} \mu^{-}$with identical spectra., which can be measured as a control. For $\left|\eta_{\tau}\right|<2.0$ the QED process has $\sigma \sim 100 \mathrm{fb}$ for $M\left(\tau^{+} \tau^{-}\right)>60 \mathrm{GeV}, 22 \mathrm{fb}$ in the $Z$-region $90 \pm 10$ $\mathrm{GeV}$ and $5 \mathrm{fb}$ in the $\mathrm{H}$ region $125 \pm 5 \mathrm{GeV} . \sigma(Z)$ is predicted to be $6-10 \mathrm{fb}[9,10]$ in $|\eta|<2$, but the branching fraction $Z \rightarrow \tau^{+} \tau^{-}$is only $3.4 \%$. The exclusive $\mathrm{H}(125)$ cross section is expected to be within a factor $\sim 3$ of $10 \mathrm{fb}$, and the branching fraction to $\tau \tau$ to be $6 \%$ (so, $18_{\div 3}^{\times 3}$ events in $100 \mathrm{fb}^{-1}$ ). Cuts on $t_{1}, t_{2}$ can enhance the signal:background, but although single arm $p+\left[H(125) \rightarrow \tau^{+} \tau^{-}\right]$is in the Stage 1 acceptance, and the background may be very small (with $n_{\text {ass. }}=0$ and kinematic contraints), the $H(125)$ signal will be small too. We have acceptance for exclusive $Z$-photoproduction with one $p$ and $Z \rightarrow e^{+} e^{-}, \mu^{+} \mu^{-}, \tau^{+} \tau^{-}(10.4 \%)$. Is it interesting? With $\gamma+\mathbb{P} \rightarrow Z$ through quark loops, we do not expect a surprise, but it does test some hard pomeron issues, as do photoproduction of $J / \psi$ and $\Upsilon$, but at higher $Q^{2}$.

I thank my colleagues in the HPS project, and the organizers of Diffraction 2012 for the opportunity to speak at a most enjoyable and useful meeting. I thank L.HarlandLang, V.A.Khoze and M.G.Ryskin for many discussions. I thank Fermilab and the U.S. Dept. of Energy for support.

\section{REFERENCES}

1. Manchester meetings, see: http://www.fp420.com/

2. M.G.Albrow and A.Rostovtsev, Searching for the Higgs boson at hadron colliders using the missing mass method, arXiv:hep-ph/0009336 (Sep. 2000); M.G.Albrow et al., Tevatron Higgs Working Group: http://arxiv.org/pdf/hep-ph/0010338.pdf (1998 contribution) FERMILAB-Pub-00/349 p.517.

3. M.G.Albrow et al., hep-ex/0511057 (March 2001).

4. V.A.Khoze, A.D.Martin, M.G.Ryskin, Eur. Phys J. C 23, 311 (2002); ibid C 19, 477 (2001).

5. M.G.Albrow et al, Quartz Cherenkov counters for fast timing: QUARTIC, J.Inst 7, P10027 (2012).

6. FP420 R\&D Project: M.G.Albrow et al., J.Inst. 4, T10001 (2009).

7. See M.G.Albrow, T.D.Coughlin, and J.R.Forshaw, Prog.Part.Nucl.Phys. 65, 149 (2010) for a review.

8. E.Chapon, C.Royon, and O.Kepcha, arXiv:0908.1061 (2009) [hep-ph].

9. L.Motyka and G.Watt, Phys. Rev. D 78, 014023 (2008).

10. A.Cisek, W.Schäfer, and A.Szczurek, Phys. Rev. 80, 074103 (2009). 